\title{
Adherence to Levothyroxine Tablet in Patients with Hypothyroidism
}

\author{
Rohan Kumar $^{1}$, Faizan Shaukat ${ }^{2}$ \\ 1. Medical Education and Simulation, Jinnah Sindh Medical University, Karachi, PAK 2. Internal \\ Medicine, Jinnah Postgraduate Medical Center, Karachi, PAK
}

$\square$ Corresponding author: Faizan Shaukat, faizan.shaukat89@gmail.com Disclosures can be found in Additional Information at the end of the article

\section{Abstract}

\section{Introduction}

Patients with hypothyroidism are managed with life-long levothyroxine (LT4) therapy. However, as with other chronic illnesses, drug adherence (DA) is a prominent issue in these patients. The aim of this study is to identify the extent of DA to LT4 in hypothyroidism patients and study the clinical factors contributing to DA in these patients.

\section{Methods}

This cross-sectional study assessed patient adherence to LT4 therapy by Modified Morisky Adherence Scale (MMAS). Factors predicting the pattern of medication adherence were also assessed in all patients. Data were entered and analyzed using SPSS v. 22.0.

\section{Results}

On MMAS, 79 (27.3\%) participants indicated low adherence, 117 (40.48\%) indicated medium adherence, and 93 (32.2\%) participants indicated high adherence. Regular endocrinologist visits and knowledge about medication were highest in high adherent patients $(\mathrm{p}<0.05)$. Need for assistance in taking medication, avoidance of medication with symptomatic relief and busy work schedule was highest in low adherent patients $(\mathrm{p}<0.05)$.

\section{Conclusion}

Patients with hypothyroidism showed moderate adherence to their treatment.

Received 04/26/2019

Review began 04/29/2019

Review ended 05/01/2019

Published 05/08/2019

\section{(c) Copyright 2019}

Kumar et al. This is an open access article distributed under the terms of the Creative Commons Attribution License CC-BY 3.0., which permits unrestricted use, distribution, and reproduction in any medium, provided the original author and source are credited.
Categories: Endocrinology/Diabetes/Metabolism, Internal Medicine, Other

Keywords: morisky medication adherence scale, hypothyroidism, levothyroxine, medication adherence, drug adherence

\section{Introduction}

Adherence (or compliance), in healthcare, is defined as the extent to which a patient is in conformity with the actual regimen prescribed by the healthcare provider. It encompasses overall behavior of the patients ranging from taking timely medicines and adjusting dietary habits to modifying their life-style [1]. In developing countries, given the scarcity of resources and inequities in healthcare system, non-adherence is somewhat comprehensible, but in developed countries only $50 \%$ adherence in patients suffering from chronic illnesses is quite alarming [2-3]. 
Although, it may be inferred that patients in clinical trials tend to behave ideally under scrutiny, leading to a false high rate of adherence; yet, only an average adherence rate is reported by clinical trials in patients with chronic diseases [4-5]. A high medical adherence rate helps reduce total healthcare costs by reducing the number of hospitalizations and emergency room visits for several common diseases [6]. Good drug adherence (DA) also leads to lower mortality rates as opposed to poor adherence [7].

A major factor contributing to adherence is 'health literacy' which is patients' ability to obtain process and understand all the information related to the progress and the complications of their disease such that they are more adherent to a treatment regimen. Improving health literacy not only involves the patients in their treatment plan, it also helps them make informed decisions about their health. This is only possible with effective communication and discussions with the healthcare providers [8]. DA has been a global issue in patients with chronic diseases. Many studies have been conducted on major chronic conditions including hypertension [8], diabetes mellitus [9], heart failure [10], and epilepsy [11].

Hypothyroidism is a chronic illness with varying prevalence in different geographical locations and a higher gender predisposition towards women [12]. Patients with hypothyroidism are managed with life-long levothyroxine (LT4) therapy, with dose adjustment according to their circulating thyroid stimulating hormone levels [13]. Missed doses of LT4 result in symptom exacerbation and can even present as a neurocognitive emergency [14]. The factors associated with missed doses or noncompliance to LT4 may be patient-related, diseases-related, or even physician-related [15]. The aim of this study is to identify patients nonadherent to their LT4 therapy and study the clinical factors contributing to DA in these patients.

\section{Materials And Methods}

This observational, cross-sectional study was conducted in the thyroid clinic of outpatient medical department of a public hospital in Karachi from July - December 2018 after ethics approval. Patients, of age $\geqslant 18$ years, who were attending the clinic with diagnosed hypothyroidism for at least one year, and could read and understand English, were included in the study after informed consent.

A self-administered performa was created which included patient gender, duration of LT4 prescription, clinical factors related to the patient, and Modified Morisky Adherence Scale (MMAS). MMAS is an 8-item relatively simple and practical tool for screening patient nonadherent to their medications in outpatient settings. It was first administered in patients with hypertension and has a reliability score of 0.83 and sensitivity of 93\% [16]. The first seven items are to be responded with a "yes" or a "no." "No" has one point and "yes" has zero points for all items. The eighth item is rated on five-point scale A-E. The response A scores one point, B scores 0.25 , C scores 0.50 , D scores 0.75 , and E scores O points. Cumulative score of MMAS-8 is $0-8$. Low adherence is indicated by MMAS $<6$, medium adherence is MMAS $6-<8$, and a score of 8 indicated high DA [17]. The clinical factors predicting pattern of DA included in this study were adapted from Shakya et al. [15].

Data were entered and analyzed using SPSS version 22 (NY:USA). Frequency and percentages were calculated for categorical data and mean and standard deviation (SD) was calculated for continuous variables. Internal consistency of the 8-item MMAS and 9-item clinical factors scale was calculated using Cronbach Alpha. Chi square was applied for comparison of categorical data. $P$ value $\leqslant 0.05$ was taken as significant.

\section{Results}

Two hundred and eighty-nine (289) individuals with diagnosed hypothyroidism participated in 


\section{Cureus}

this study. There were 201 (69.55\%) women and 88 (30.44\%) men. There were 140 (48.4\%) participants who had been prescribed LT4 for more than 5 years, and 149 (51.55\%) participants who had been prescribed LT4 for 1-5 years.

The internal consistency of MMAS in this study was 0.80 and that of the clinical factors scale was 0.75 . On MMAS, 79 (27.3\%) participants scored $<6$ and were indicated to have low DA, 117 (40.48\%) scored $6-<8$ and were indicated to have medium DA, and 93 (32.2\%) participants scored 9 indicating high DA. The distribution of extent of DA and its relationship with patient gender and duration of prescription are shown in Table 1.

\begin{tabular}{|c|c|c|c|c|c|c|c|}
\hline \multirow{2}{*}{$\begin{array}{l}\text { Level of drug } \\
\text { adherence }\end{array}$} & \multirow{2}{*}{$\begin{array}{l}\text { Total sample } \\
(n=289)\end{array}$} & \multicolumn{3}{|l|}{ Gender } & \multicolumn{3}{|c|}{ Duration of LT4 prescription } \\
\hline & & $\begin{array}{l}\text { Male } \\
(n=88)\end{array}$ & $\begin{array}{l}\text { Female } \\
(n=201)\end{array}$ & $\begin{array}{l}\mathrm{P} \\
\text { value }\end{array}$ & $\begin{array}{l}1-5 \text { years } \\
(n=149)\end{array}$ & $\begin{array}{l}>5 \text { years } \\
(n=140)\end{array}$ & $P$ value \\
\hline High DA & $93(32.2 \%)$ & $\begin{array}{l}43 \\
(48.8 \%)\end{array}$ & $50(24.8 \%)$ & & $28(18.7 \%)$ & $65(46.4 \%)$ & \\
\hline Medium DA & $117(40.5 \%)$ & $\begin{array}{l}29 \\
(32.9 \%)\end{array}$ & $88(43.7 \%)$ & 0.0002 & $87(58.3 \%)$ & $30(21.4 \%)$ & $<0.00001$ \\
\hline Low DA & $79(27.3 \%)$ & $\begin{array}{l}16 \\
(18.2 \%)\end{array}$ & $63(31.3 \%)$ & & $34(22.8 \%)$ & $45(32.1 \%)$ & \\
\hline
\end{tabular}

\section{TABLE 1: Relationship of level of drug adherence with patient gender and duration of \\ LT4 prescription}

DA, drug adherence; LT4, levothyroxine

The patients' DA levels were then correlated with clinical factors related to the disease. These are summarized in table 2. Among individuals with high DA patients, 72 (77.4\%) visited their doctors regularly, 45 (48.3\%) were educated about their disease and 68 (73.1\%) about their medication, 55 (59.2\%) could afford their medicines, medicines were accessible to 52 (55.9\%), 25 (26.8\%) needed assistance in taking medications and had busy work schedule each, 14 (15.1\%) missed doses when symptoms alleviated, and 11 (11.8\%) missed doses due to side effects. The responses on education about the disease and the medications, regular endocrinologist visits, medication affordability, work schedule, and need of assistance were significantly correlated with the level of DA as shown in Table 2.

\section{Clinical factors related to the disease}

$$
\text { High DA }(n=93)
$$

Medium DA (n=117)

Low DA ( $n=79)$

$P$ value

Regularly visit endocrinologist

Yes

No

Knowledge given about the condition by the managing endocrinologist

Yes

$45(48.3 \%)$

$72(61.5 \%)$
25 (31.6\%)

$54(68.3 \%)$ 


\section{Cureus}

No

$48(51.6 \%)$

$45(38.4 \%)$

$42(53.1 \%)$

Knowledge given about the medication by the managing endocrinologist

$\begin{array}{llll}\text { Yes } & 68(73.1 \%) & 69(58.9 \%) & 24(30.3 \%)< \\ \text { No } & 25(26.8 \%) & 48(41.0 \%) & 55(69.6 \%)\end{array}$

Need assistance taking medication

Yes

$25(26.8 \%)$

$34(29.1 \%)$

$48(60.7 \%)$

$68(73.1 \%)$

$83(70.9 \%)$

$<0.00001$

No

Medication affordability

Yes

$55(59.2 \%)$

$84(71.7 \%)$

$29(36.7 \%)$

$38(40.8 \%)$

$33(28.2 \%)$

$<0.00001$

No

Medication accessibility

Yes

52 (55.9\%)

$67(57.2 \%)$

$35(44.3 \%)$

$41(44.1 \%)$

$50(42.7 \%)$

$44(55.7 \%)$

0.16

No

Busy work schedule

Yes

$25(26.8 \%)$

$53(45.3 \%)$

$46(58.2 \%)$

$68(73.1 \%)$

$64(54.7 \%)$

0.0001

No

Avoid medication when symptomatic relief

Yes

$14(15.1 \%)$

$41(35.1 \%)$

$60(75.9 \%)$

$79(84.9 \%)$

$76(64.9 \%)$

$19(24.1 \%)$

$<0.00001$

Avoid medication due to side affects

Yes

$11(11.8 \%)$

$19(16.2 \%)$

$20(25.3 \%)$

No

$82(88.2 \%)$

$98(83.7 \%)$

\section{TABLE 2: Impact of disease-related clinical factors on the extent of drug adherence}

DA, drug adherence

\section{Discussion}

More than two-third individuals with hypothyroidism are medium-to-high adherent to their prescriptions. More men were high adherent and more women were moderate-to-low adherent. Patients with 1-5 years of LT4 prescription were moderately adherent and patients with longer duration of prescription showed high adherence. It was seen that patient education about the disease and the medications, regular visits to the endocrinologist, medication affordability, work schedule, and assisted medication taking were significantly correlated with the level of 
DA. Medication side effects and accessibility was not significantly correlated to extent of DA.

To the best of our knowledge, this study is primary in reporting the extent of medication compliance among hypothyroid patients in Pakistan. Although, this is a single-center study and cannot be generalized to the situation prevailing in the country, still, it provides a very concrete picture as this hospital is the largest public tertiary care in the entire province. An important limitation to this study is that the DA rate was self-reported and not checked or confirmed via number of empty pill leaflets, drug card, or any other method.

In an observational cross-sectional study, conducted in 320 patients in north-eastern Italy [18], the study population had $1.9 \%$ low adherers (compared to $27.3 \%$ in our study), $10.9 \%$ medium adherers (compared to $40.5 \%$ in ours), and $87.2 \%$ high adherers (as opposed to $32.2 \%$ in this study). However, this study also showed a higher adherence rate in patients taking liquid LT4 as compared to tablet LT4, as patients had difficulty remembering and sticking to the treatment plan; a factor not taken in consideration as it is not yet available in Pakistan. A lower adherence rate was reported by a study in Lebanon, with significant improvements in adherence with good doctor-patient communication and regular appointments; as opposed to last minute appointment cancellations, patients with history of water pipes and alcohol intake per week [19]. A multinational, cross-sectional, internet-based study, conducted in 2016 [20], gathered data from pregnant women in 18 countries. The adherence rate was low in $17 \%$ while it was reported to be moderate and high in 44\% and 39\%, respectively. Younger age, unstable relationships and no folic acid intake were associated with lower adherence. In Hepp Z et al. [21], lower all-cause and hypothyroidism-related costs and resource utilization are reported in adherers as compared to non-adherers and a lower incidence of concurrent co-morbid diagnosis implying that compliance leads to both clinical and economical benefits in the long run.

From all data and literature, one common conclusion which can be drawn is, that healthcare professionals play a significant role in the management of hypothyroidism and can help reduce the barriers to optimal treatment by patient education about the drugs and their disease. Furthermore, involving pharmacists and nurses can help increase thyroid replacement in patients. Physicians should take into consideration the financial and social conditions, relationship status and mobility of a patient while prescribing medications. A keener emphasis on diet and addressing issues like sexual dysfunction (otherwise considered a taboo in Pakistani society), weight gain and lethargy in concerned patients helps build a better doctor-patient relationship and develops patient confidence in their healthcare provider.

\section{Conclusions}

Hypothyroidism is a chronic progressive medical illness which demands strict compliance to medication for disease control. Like other chronic illnesses, patients with hypothyroidism also have difficulty in maintaining compliance to their treatment. Patients with hypothyroidism showed moderate adherence to their treatment. Patient education, regular endocrinologist visits, and affordability of medications increased medication compliance. Improving medication compliance demands a multi-faceted approach with robust efforts from both the health practitioner and also the patient. Health professionals should be willing to develop and implement potentially effective means to achieve this.

\section{Additional Information \\ Disclosures}

Human subjects: Consent was obtained by all participants in this study. Jinnah Sindh Medical University Ethical Review Board issued approval MU/ECA/11/023. Animal subjects: All authors 
have confirmed that this study did not involve animal subjects or tissue. Conflicts of interest: In compliance with the ICMJE uniform disclosure form, all authors declare the following:

Payment/services info: All authors have declared that no financial support was received from any organization for the submitted work. Financial relationships: All authors have declared that they have no financial relationships at present or within the previous three years with any organizations that might have an interest in the submitted work. Other relationships: All authors have declared that there are no other relationships or activities that could appear to have influenced the submitted work.

\section{References}

1. Lieber SR, Helcer J, Shemesh E: Monitoring drug adherence. Transplant Rev. 2015, 29:73-7. 10.1016/j.trre.2014.10.001

2. Sabaté E: Adherence To Long-Term Therapies: Evidence for action . WHO publications, Geneva, Switzerland; 2003.

3. Nieuwlaat R, Wilczynski N, Navarro T, et al.: Interventions for helping patients to follow prescriptions for medications. Cochrane Database Syst Rev. 2014, 11:10.1002/14651858.CD000011.pub4

4. Cramer J, Rosenheck R, Kirk G, et al.: Medication compliance feedback and monitoring in a clinical trial: predictors and outcomes. Value Health. 2003, 6:566-73. 10.1046/j.15244733.2003.65269.x

5. Sokol MC, McGuigan KA, Verbrugge RR, Epstein RS: Impact of medication adherence on hospitalization risk and healthcare cost. Med Care. 2005, 43:521-30.

6. Simpson SH, Eurich DT, Majumdar SR, et al.: A meta-analysis of the association between adherence to drug therapy and mortality. BMJ. 2006, 333:15. 10.1136/bmj.38875.675486.55

7. Miller TA: Health literacy and adherence to medical treatment in chronic and acute illness: a meta-analysis. Patient Educ Couns. 2016, 99:1079-86. 10.1016/j.pec.2016.01.020

8. Vrijens B, Antoniou S, Burnier M, de la Sierra A, Volpe M: Current situation of medication adherence in hypertension. Front Pharmacol. 2017, 8:100. 10.3389/fphar.2017.00100

9. Guénette L, Moisan J, Breton MC, Sirois C, Grégoire JP: Difficulty adhering to antidiabetic treatment: factors associated with persistence and compliance. Diabetes Metab. 2013, 39:2507. 10.1016/j.diabet.2012.12.005

10. Krueger K, Botermann L, Schorr SG, Griese-Mammen N, Laufs U, Schulz M: Age-related medication adherence in patients with chronic heart failure: a systematic literature review. Int J Cardiol. 2015, 184:728-35. 10.1016/j.ijcard.2015.03.042

11. Dash D, Sebastian TM, Aggarwal M, Tripathi M: Impact of health education on drug adherence and self-care in people with epilepsy with low education. Epilepsy Behav. 2015, 44:213-7. 10.1016/j.yebeh.2014.12.030

12. Taylor PN, Albrecht D, Scholz A, et al.: Global epidemiology of hyperthyroidism and hypothyroidism. Nat Rev Endocrinol. 2018, 10.1038/nrendo.2018.18

13. Hennessey JV, Espaillat R: Current evidence for the treatment of hypothyroidism with levothyroxine/levotriiodothyronine combination therapy versus levothyroxine monotherapy. Intl J Clin Pract. 2018, 72:e13062. 10.1111/ijcp.13062

14. Kandukuri RC, Khan MA, Soltys SM: Nonadherence to medication in hypothyroidism: a case report. Prim Care Companion J Clin Psychiatry. 2010, 12: 10.4088/PCC.09m00863gre

15. Shakya Shrestha S, Risal K, Shrestha R, Bhatta RD: Medication adherence to levothyroxine therapy among hypothyroid patients and their clinical outcomes with special reference to thyroid function parameters. Kathmandu Univ Med J. 2018, 62:129-37.

16. Morisky DE, Ang A, Krousel-Wood M, Ward HJ: Predictive validity of a medication adherence measure in an outpatient setting. J Clin Hypertens. 2008, 10:348-54. 10.1111/j.17517176.2008.07572.x

17. Okello S, Nasasira B, Muiru AN, Muyingo A: Validity and reliability of a self-reported measure of antihypertensive medication adherence in Uganda. PloS one. 2016, 1:0158499.

10.1371/journal.pone.0158499

18. Cappelli C, Castello R, Marini F, et al.: Adherence to levothyroxine treatment among patients with hypothyroidism: a Northeastern Italian Survey. Front Endocrinol. 2018, 9:699.

10.3389/fendo.2018.00699 


\section{Cureus}

19. El Helou S, Hallit S, Awada S, et al.: Adherence to levothyroxine among patients with hypothyroidism in Lebanon. World Health Organization. 2018,

20. Juch H, Lupattelli A, Ystrom E, Verheyen S, Nordeng H: Medication adherence among pregnant women with hypothyroidism-missed opportunities to improve reproductive health? A cross-sectional, web-based study. Patient Educ Couns. 2016, 99:1699-707. 10.1016/j.pec.2016.04.006

21. Hepp Z, Lage MJ, Espaillat R, Gossain VV: The association between adherence to levothyroxine and economic and clinical outcomes in patients with hypothyroidism in the US. J Med Econ. 2018, 21:912-9. 10.1080/13696998.2018.1484749 